\title{
Model Pad Geometry Analysis
}

\author{
Tamara Evgenevna Ilina ${ }^{1}$, Nikolay Vasilevich $\operatorname{Prodan}^{1} \&$ Pavel Viktorovich Bulat ${ }^{1}$ \\ ${ }^{1}$ ITMO University, Russia \\ Correspondence: Tamara Evgenevna Ilina, ITMO University, 197101, Saint Petersburg, Russia. E-mail: \\ tamara-190@yandex.ru
}

Received: November 19, 2014

Accepted: November 24, $2014 \quad$ Online Published: August 30, 2015

doi:10.5539/mas.v9n9p322

URL: http://dx.doi.org/10.5539/mas.v9n9p322

\begin{abstract}
For gas-lubricated bearings the form of a lubricating clearance in many respects determines the carrying capacity value of the bearing. Furthermore, the parameters defining this form have, for the most part, the optimal values.

Today, the most typical for computation of a gas base layer is use of software with solvers which contain equations of the gas lubrication theory based on the Reynolds equation. However, the total computation of the system of gas feed into the gas-static bearing box in such setting meets methodical complications: in the gas feed system turbulent flows appear, and their computation is necessary to be carried out in the full Navier-Stokes equations with connection of the applicable model of turbulence, and there is a laminar flow in the lubricating film. The solution of the computation problem of the gas base layer in the Navier-Stokes full equations is considered. The method was verified with use of known self-similar solutions. Based on the tried-and-true method, parametric study of a pad with simple plain bores, a pad with a slotted nozzle and composite pads with angular clearances designed under the Rayleigh principle were carried out.
\end{abstract}

Keywords: gas-static bearing, pads, carrying capacity, gas base layer, navier-stokes equations, rayleigh profile

\section{Introduction}

It is the purpose of this work to study the carrying capacity of model pads of a gas-static bearing which principle of operation is based on creation of a lift force with air injection by an external device.

When designing and optimizing gas bearings, the engineers have to solve a problem of choice of a method and means of gas bearing modeling. To the present, the basic physical properties of gas-static bearings have been well learnt, the basic characteristic dependences are specified.

Transition to oil-free transmissions using gas bearings, first of all, is connected to increase of efficiency of means of aerospace engineering, engines, gas-turbine power units, turbo-refrigerator units.

The works on usage of gas lubrication in high-velocity turbomachines have been carried out since the early 1950s. In the USSR and today Russia these works have been carried out in Bauman MSTU, Geliymash NPO, Kriogenmash NPO, NAUKA NPO, MAI, and DSU. Abroad such works are conducted in Maxi-Garret AiResearch (Allied Signal), General Electric, MTI, United Technologies Sof., MITI (USA) Air Liquid, ABG Semka (France), British Oxygen (Great Britain) etc.

The basic works of Scheinberg (1979) and Constantinescu (1963), Rippel (1967), Gressem and Powell (1966) are dedicated to the problem of gas-lubricated bearings. We also should mark the works of Kotlyar (1967), Zabolotsky (1961, 1970), Loitsansky and Stepanyants $(1958,1967)$. The detailed historical review of the works dedicated to gas-lubricated bearings is given in the work of Bulat M.P., Bulat P.V. (2013).

A distinction is made among gas-static, gas-dynamic and hybrid bearings. You will find the detailed classification in the work of Bulat M.P., Bulat P.V. (2013).

The undertaken studies (Kosmynin A.V. et al., 2011; Sipenkov I.E., 1968; Frolov K.V., 2008) demonstrate that even rather small deviations of the parameters values from the optimal values result in considerable decrease of carrying capacity of the bearing or to its complete inoperativeness.

By the experience of designing and applying of bearings with external boosting it is known that we have to tend to rather small diameters of feeding nozzles (less than $0.1 \mathrm{~mm}$ ). Tending to small diameters of nozzles is dictated by the necessity of receiving of acceptable gas consumption through the bearing. The less the diameter of 
openings and the more their amount, the more the stiffness of a lubricating film . The stiffness is understood in the same sense as for a spring. This circumstance led the designers and researches to a concept of application of porous liners in gas-static bearings.

Firstly, the porous radial bearings with external gas boosting were described in the work of Robinson and Sterri (1958). The studies showed that the porous gas bearings with external boosting posses, other factors being equal, heightened stiffness of a lubricating film, the best carrying and damping capacity, they are less inclined to loss of stability as compared with different constructions of gas bearings.

Despite of the indicated advantages, the bearings with porous liners have the following defects:

- change of the gas permeability of machined porous materials as a result of plastic deformation of their surface layer;

- the bearings with porous liners are unstable in sizes, the high air rate, and from here heightened power consumption for compressed air supply are typical for them.

At this stage, one of the key problems is development of such geometry of the supply system and feeding nozzles which provides the stiffness and economy of gas-static bearing closer to the characteristics of the bearing with porous liners, with significantly fewer nozzles.

The analysis of the gas bearing study results demonstrates that, despite of considerable success in this field, the problems related to the basic defects of gas bearings (small carrying and damping capacities of the gaslubricating film, instability of work) remain actual. For gas-lubricated bearings the form of a lubricating clearance, to a large extent, determines the value of the bearing carrying capacity, moreover, the parameters defining this form have, in their majority, the best values.

\section{Method}

\subsection{Problem Statement}

The theory of hydrodynamic lubrication (Rayleigh, 1918) covers the fact that the carrying capacity of carrier of pads is defined by the working gas rate. For a solution of the direct problem (definition of carrying capacity of the bearing according to the given geometry of the lubricating film), the pad should be optimized at a constant given working gas rate. In this case, the optimal pad is that one which for the given rate will supply the maximum carrying capacity.

In case of an inverse problem (definition of the geometry or separate geometrical parameters of the lubricating film, on the basis of the given mechanical characteristics of the bearing), the optimal one is a pad which provides the minimum rate for the given carrying capacity.

This article gives solutions of direct problems for pads with 3 cylindrical nozzles, with a simple slotted opening and for pads with the complicated form of the lubricating film.

The article provides a research of streams realized in the bearing gas layer, a stream in the feeding system is considered as given. For the problem simplification the pad bearing surface was developed on the plain. Such an approach is quite allowable, since the lubricating film thickness is third-order smallness as compared to the pad characteristic dimension. The problems were solved in laminar setting.

\subsection{Research Technique}

The basic source of difficulties in the analysis of bearings with external boosting is the availability in the feeding system of two gas flow modes: laminar mode and turbulent mode. So, by virtue of smallness of the lubricating film thicknesses, as compared to characteristic dimensions of the bearing, the stream in the lubricating layer is considered as laminar. In the gas feeding system, the stream is turbulent (Fig. 1a). In Fig. 1b, you can find under the central nozzle the availability of supersonic efflux velocities. Such efflux modes can result in lock-out of a part of the opening and the onset of oscillations. The interaction of a supersonic jet and a barrier is described in the work of Ginsburg I., Sokol E., Uskov V. (1976). 


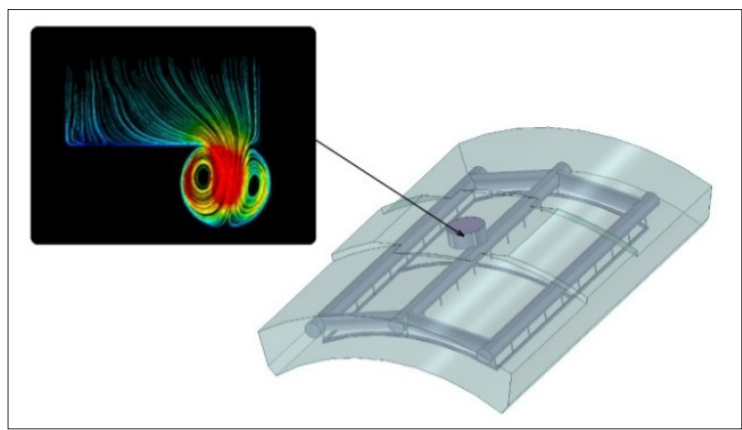

a)

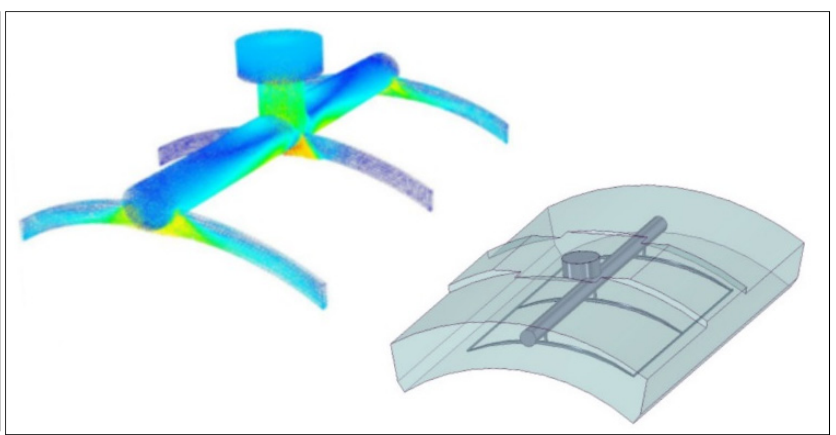

b)

Figure 1. Gas flow in the feeding system: a) Vortex flow formation; b) Irregular velocity field for a pad with 3 crescent grooves

It is necessary to model the vortex flows based on the Navier-Stokes full equations with use of the turbulence model, in this case Transition SST based on two transport equations, one of which is for intermittency, and the other one is for the pressure criteria in terms of the Reynolds number calculated for the momentum thickness. The Transition SST model allows more precisely describing the turbulence at the expense of introduction of additional transport equations and shows good results for computation of near-wall regions.

The gas lubrication Reynolds equations (Reynolds O., 1886), which are included in standard packages like Ansys CFX, for a laminar flow in the lubricating film do not allow joining solutions on the nozzle boarder.

To provide a reliable solution joint in the lubricating film, it is necessary to use the Navier-Stokes equations, similarly to the feeding system, in the lubricating film, only without connection of the turbulence models. The Navier-Stokes equations allow taking into account the near-critical flows formed when flowing out of the orifices, viscous-inviscid interaction and transient processes in the lubricating film.

Package ANSYS Fluent was used as a tool for the problem solution. The package solver uses the Navier-Stokes equations.

$$
\begin{gathered}
\frac{\partial \rho \mathbf{v}}{\partial t}+\nabla(\rho \mathbf{v} \cdot \mathbf{v})=-\nabla \mathrm{p}+\nabla(\boldsymbol{\tau})+\mathbf{F} \\
\frac{\partial \rho}{\partial t}+\nabla(\rho \mathbf{v})=0
\end{gathered}
$$

where $\mathbf{v}$ is the fluid velocity vector, $\tau$ - viscous stress tensor, $\mathbf{F}$ - vector of volume forces, $\mathbf{p}$ - static pressure, $\rho-$ density.

The components of the viscous stress tensor $\tau_{\mathrm{ij}}$ is defined as

$$
\tau_{i j}=\mu\left(\frac{\partial u_{i}}{\partial x_{j}}+\frac{\partial u_{j}}{\partial x_{i}}-\frac{2}{3} \delta_{i j} \frac{\partial u_{k}}{\partial x_{k}}\right),
$$

where $\mu$ is the dynamic (molecular) viscosity, $\mathrm{u}_{\mathrm{ij}}$ - components of the velocity vector, $\delta_{\mathrm{ij}}$ - Kronecker symbol.

It is necessary to specify the initial and boundary conditions for the equation solution.

The initial velocity distribution which meets the continuity equation is set as the initial conditions, as well as the initial temperature and pressure distribution.

The boundary conditions for computation are reduced to the following setting:

- conditions of adherence on the bearing walls;

- $\quad$ pressure of streamline flow on the bearing edges (1 atm);

- $\quad$ symmetry conditions meeting a full-coverage (non-dissected) bearing (a fictitious layer of difference cells is formed on the border, with a velocity equal in the value and opposite in the direction to the velocity vectors in the border cells);

- constant rate on the nozzles. 
The solution result specifies the resultant vector of pressure forces in the lubricating film and pressure visual functions.

The general technique of checking computation is given in the work of Beschastny V. (2005).

\subsection{Numerical Technique Verification}

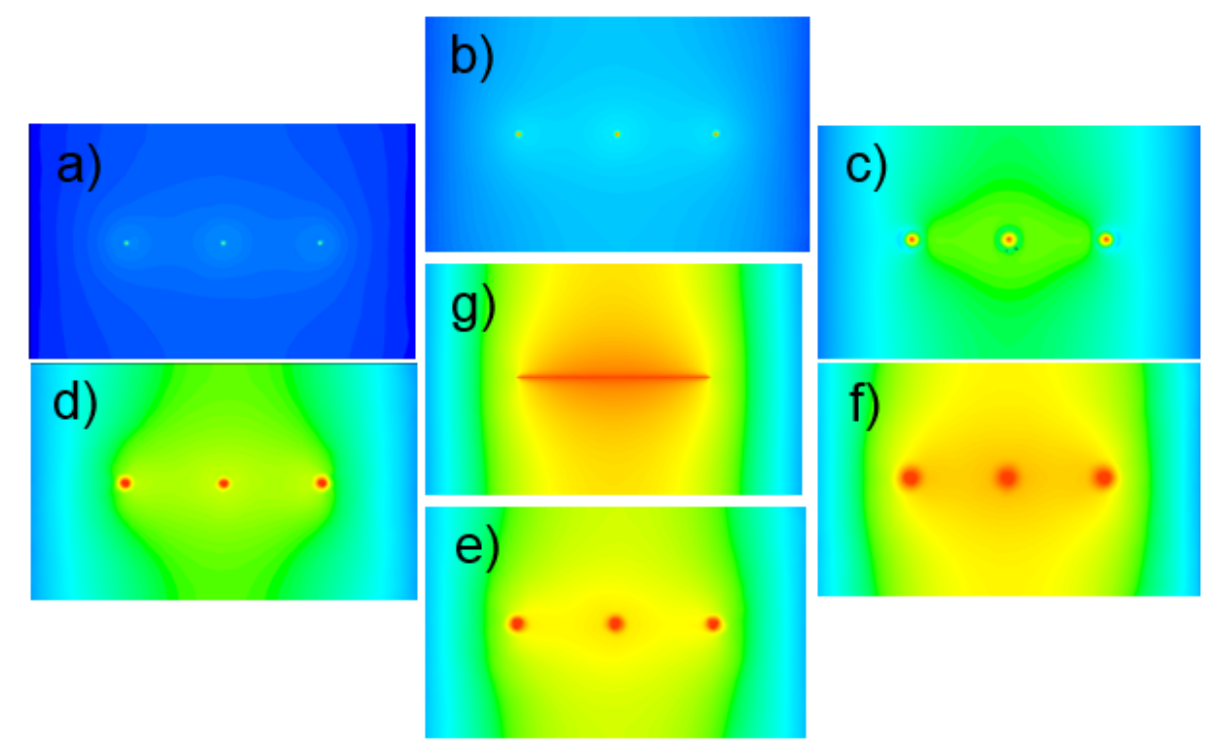

Figure 2. Pressure distribution on the shaft with increase in the hole diameters of the feeding system of working substance, comparison with the slotted nozzle: a) $d=0.5 \mathrm{~mm}$; b) $d=1 \mathrm{~mm}$; c) $d=2 \mathrm{~mm}$; d) $d=3 \mathrm{~mm}$; e) $d=4 \mathrm{~mm}$; f) $\mathrm{d}=5 \mathrm{~mm} ; \mathrm{g}) \mathrm{h}=0.35 \mathrm{~mm}$

The computation results demonstrated (Fig. 2) that the pressure isolines completely repeat the self-similar solutions given in the book of в книге Constantinescu (1963). Fig. 2 demonstrates the pressure fields for 6 pads with holes of different diameters, and 1 pad with a slotted nozzle. The gas flows out of the clearance into the environment through two vertical sides of the pad.

In such a way, it is possible to conclude that this technique is applicable for study of parameter distribution in the lubricating film of a gas-static bearing.

We know that lifting capacity linearly depends on the working gas rate. This fact is used for testing the lifting capacity computation technique. Let's evaluate the diameter influence on a pad with 3 simple parallel holes. Let's consider pads with 3 different diameters of holes: 1,3 and $5 \mathrm{~mm}$.

For the direct problem solution, the pad lifting capacity is compared for the given rate of working gas: $2 ; 4 ; 6$ and $9 \mathrm{~g} / \mathrm{s}$.

The test results is shown in Fig. 3a. The diagram uses a logarithmic scale for lifting capacity values mapping. The similar computation was carried out for pads with a simple slotted orifice of different width: $0.04,0.35$ and $0.98 \mathrm{~mm}$. The results are given in Fig. $3 \mathrm{~b}$. 


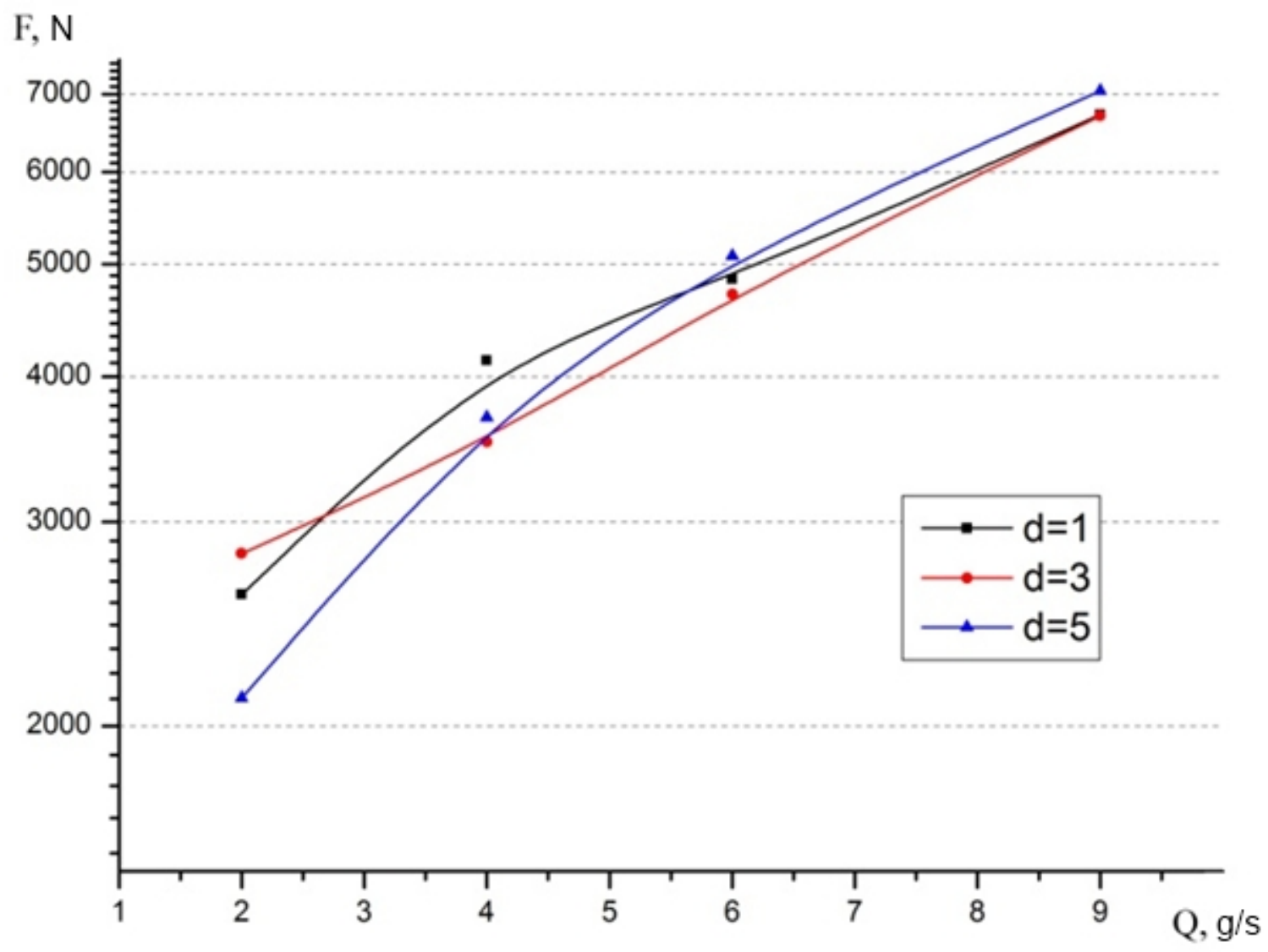

a) 


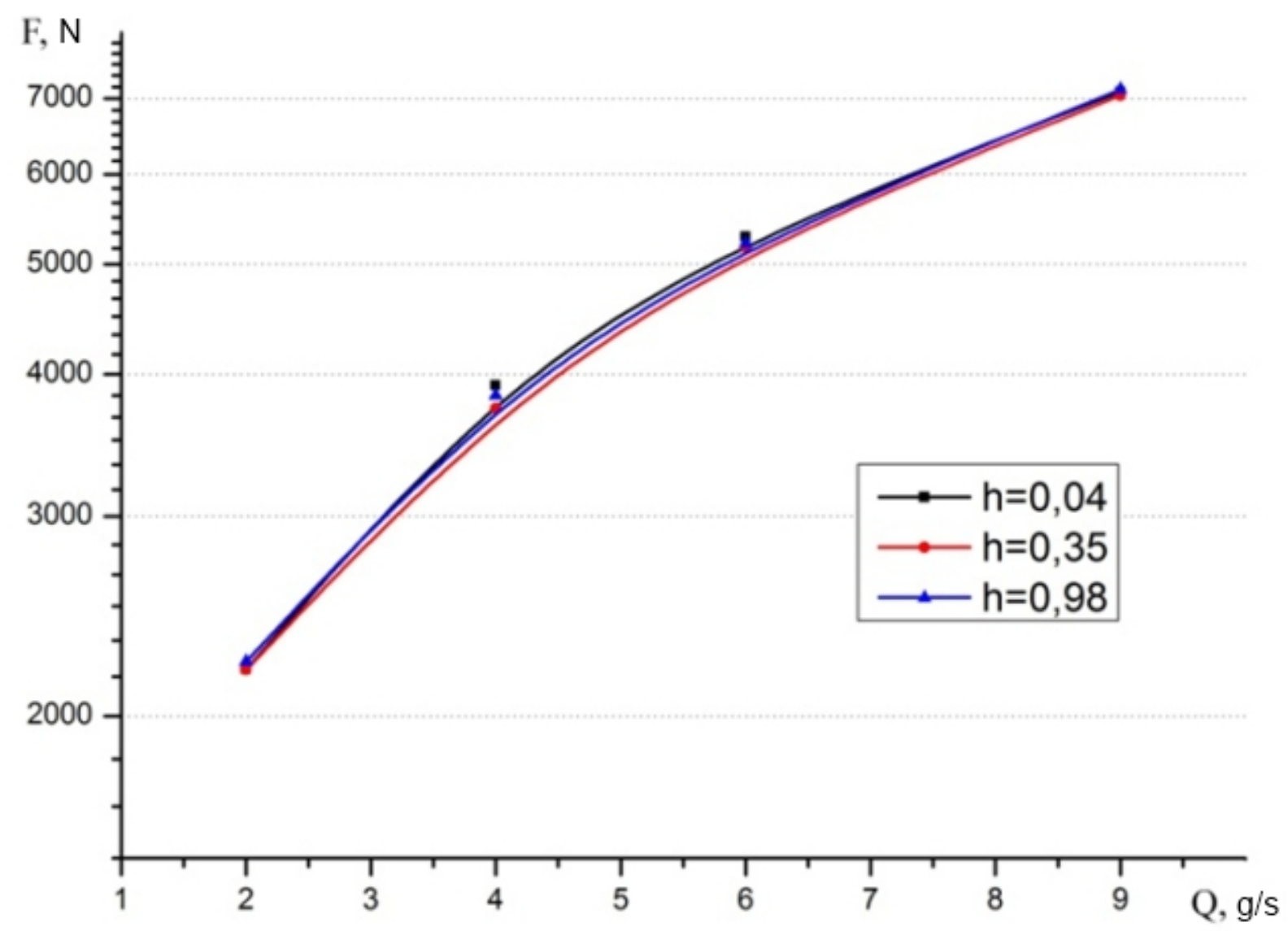

b)

Figure 3. Dependence of the lifting capacity on the set rate of working gas a) for a pad with 3 holes of different diameters, $b$ ) for a pad with 1slotted orifice of different width

The results demonstrate computation of the approximate linear dependence of increase of lifting capacity along with increase of working gas rate. The curves of dependence of lifting capacity on the rate for a pad with 3 holes for different diameters are located non-parallel - they cross, which indicates the fact that for the set lifting capacity there is a pad with the optimal diameter of holes providing the minimum rate.

\section{Results}

\subsection{Evaluation of Influence of the Lubricating Film Thickness}

Let's evaluate the influence of the lubricating film thickness on the pad lifting capacity. Direct problem solution results in the obtained values of lifting capacity for pads of a gas-static bearing with parallel holes of different diameters and the thickness of lubricating film: 10, 20, 30, 40 and $50 \mathrm{mcm}$ (Fig. 4). Computation was conducted for the set constant rate of working gas $5 \mathrm{~g} / \mathrm{s}$.

The curve angle meets the lubricating film stiffness.

Within the range $10-20 \mathrm{mcm}$ the dependence of lifting capacity on the lubricating film thickness is approximate linear, for minor change of the lubricating film thickness you can find strong change of the pad lifting capacity, i.e. the stiffness is high. In this case, the lubrication film works like a spring. Within the range $30-50 \mathrm{mcm}$ and more, it is possible to characterize by the lubricating film constant stiffness, but its absolute value is less. The area $20-30 \mathrm{mcm}$ can be called as a dead space, as the lifting capacity doesn't change practically along the thickness change, the stiffness is minimum. The pressure drop in the given area is such that in the feeding nozzles a shock-wave arises and locks them. This process lasts until the force acting on the pad reaches a threshold value which is capable to push the wave inside the system. 
In the given zone there can be dangerous vibration of the pad, since the damping ratio of the lubricating film is smallest, and gas makes no resistance to a vertical displacement of the shaft relative to the pad. Such behavior of a lubricating film is called as loss of stability of a lubricating film.

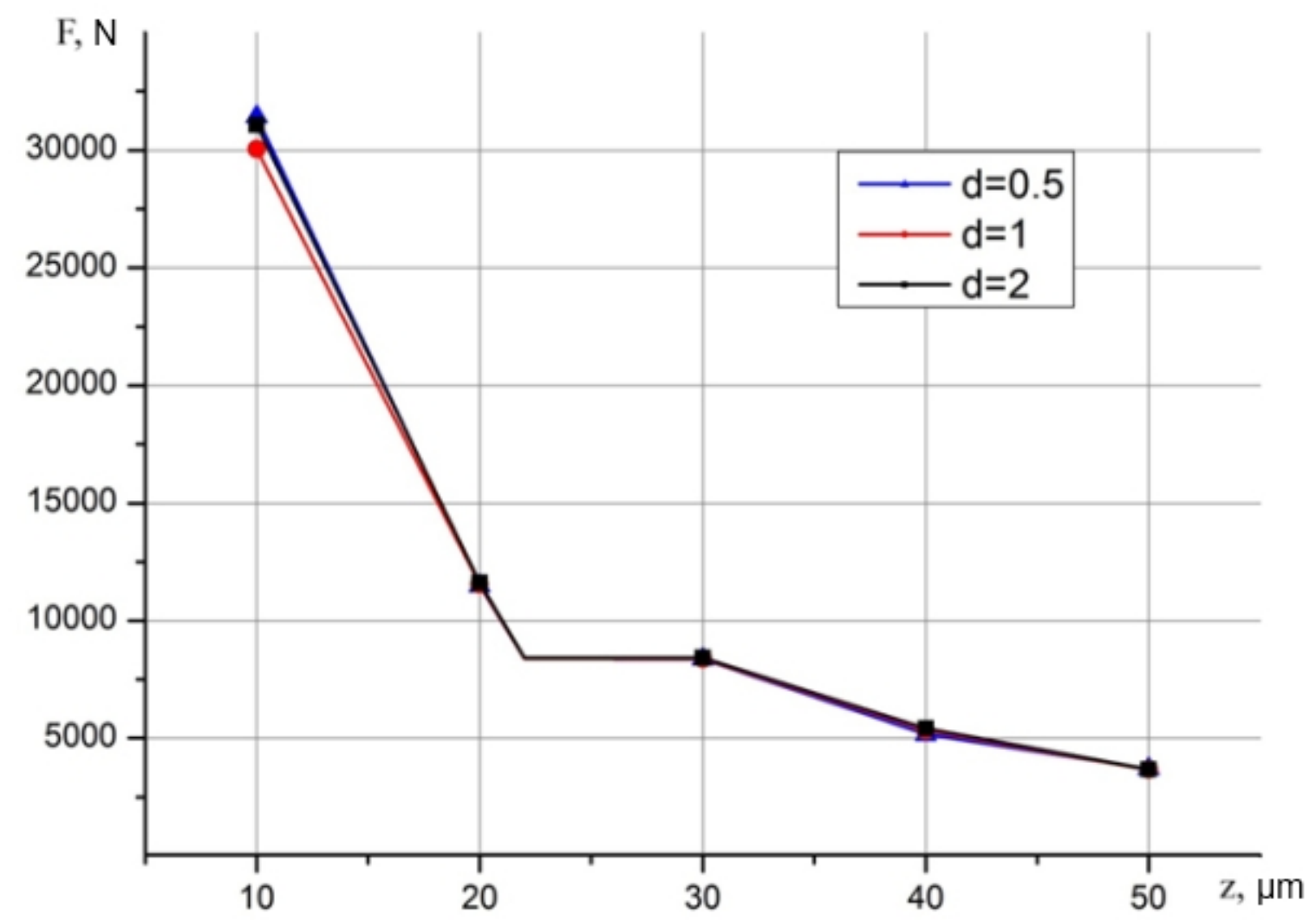

Figure 4. Dependence of lifting capacity on the lubricating film thickness for a simple pad with 3 parallel holes. Working gas rate is $5 \mathrm{~g} / \mathrm{s}$

\subsection{Computation of Pads with a Shaped Lubricating Film}

The work of Rayleigh on the theory of gas lubrication (Rayleigh, 1918) shows that for noncompressible lubricant, the optimal profile, from the point of view of lifting capacity, is a single-stage piecewise constant profile. In accordance with this theory two gas-static pads were developed: a pad with simple parallel holes with angular clearances located along the influence lines and a pad with the Rayleigh angular clearances (Fig. 5). 


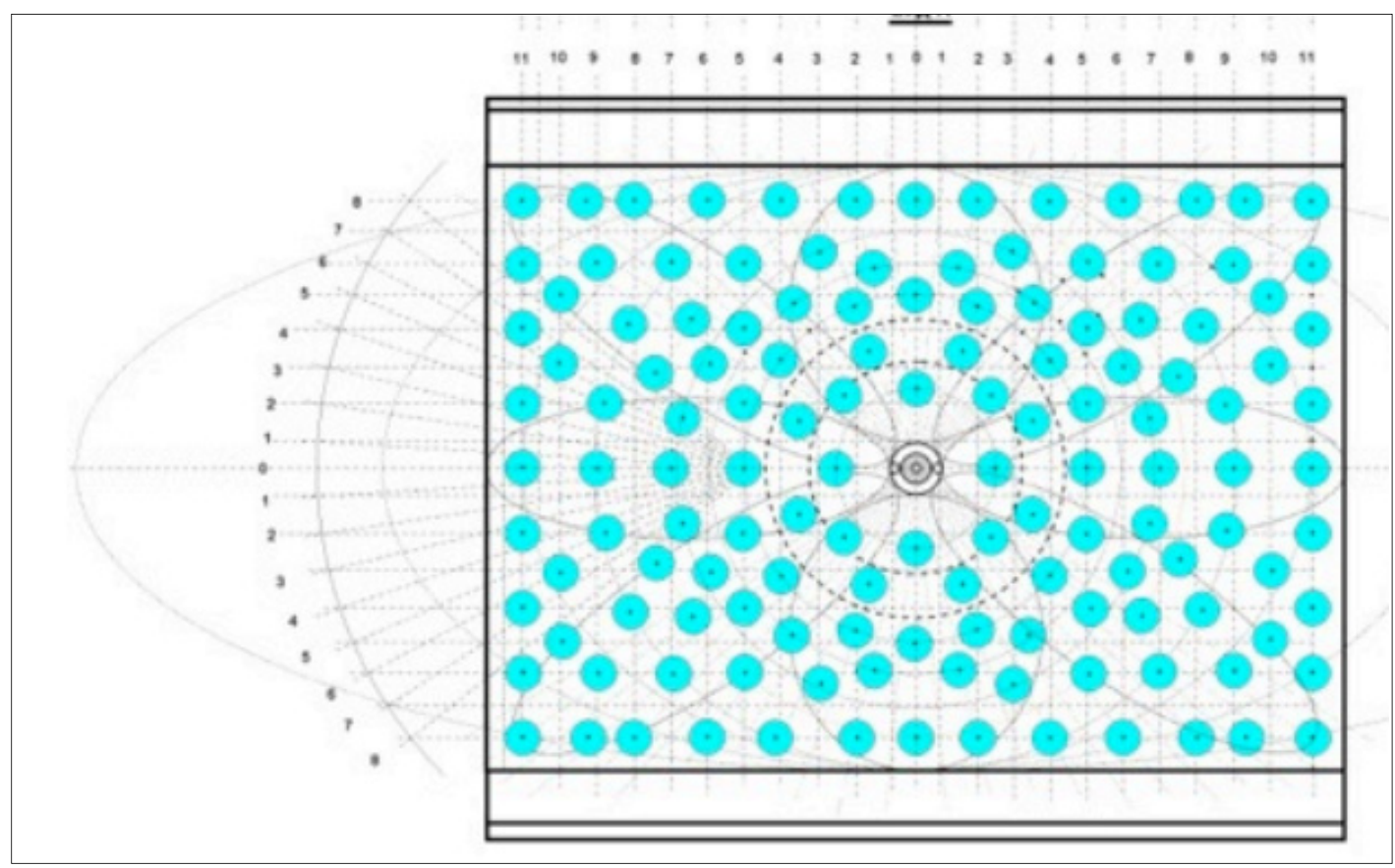

a)

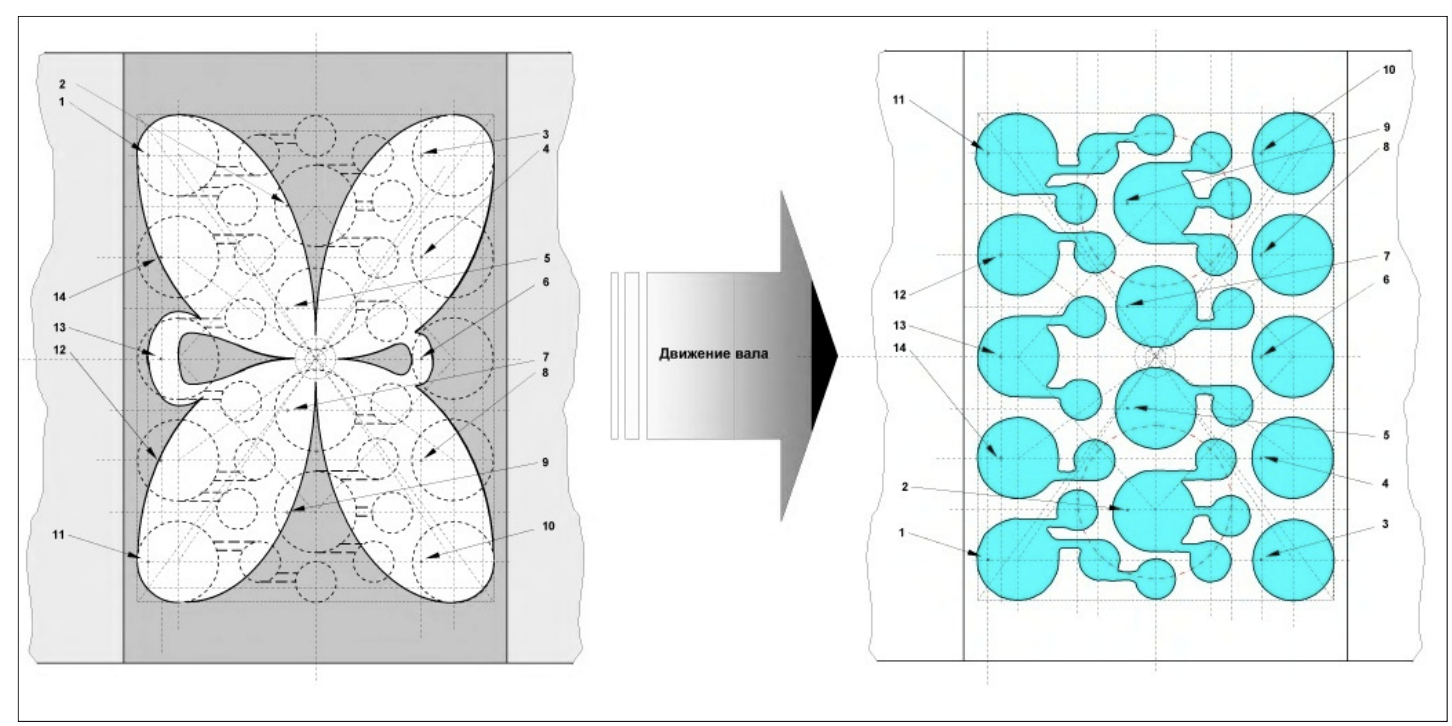

b)

Figure 5. a) a pad with simple parallel holes with angular clearances located along the influence lines; b) a pad with the Rayleigh angular clearances (arrows 1-14 show the nozzles position)

A pad with simple parallel holes with angular clearances located along the influence lines (Fig. 5a) is designed based on the principle of porous pads: a number of feeding nozzles by small diameter $0.5 \mathrm{~mm}$. Holes are located according to the sectors. An angular clearance, a cylindrical hollow by thickness of $1 / 3$ lubricating film, is stipulated under each nozzle. In accordance with the Rayleigh principle, such a solution must increase the lifting capacity. Air is injected into the pad through the central hole and then distributed in the hollow on the external side of the support. From there air goes in the feeding nozzles. Location of holes pursues a problem to achieve the maximum even distribution of pressure on the base surface.

A pad with the Rayleigh angular clearances (Fig. 5b) represents the base surface with nozzles located according to the diagram in Fig. $5 b$ (nozzles are indicated with arrows). Total of feeding nozzles is 14 . Under the feeding 
nozzle, as well as in a pad with simple parallel holes with angular clearances located on the influence lines there is a angular clearance stipulated. A hollow in the pad 2 is shaped and consists of communicating cavities of different diameters. Air from the feeder flows at first to the hollow of large diameter, and then along thin channels it flows into the smaller hollow. The disposition of hollows is asymmetrical relatively the shaft rotation axis, since it takes into account the direction of rotation. On the pad external surface there is a cavity in the form of butterfly, which is fed with the working gas. Such geometry of hollow provides an even distribution of gas in the gas feeding system for the lubricating film, and also equal pressures on the nozzles.

In such a way 2 pads were developed, one of which corresponds to the model of a porous pad, and the other has considerably smaller amount of openings, but they are of bigger diameter. Let's define their lifting capacity.

For the pad computation, the constant rate of working gas through the nozzles was set equal to $12.5 \mathrm{~g} / \mathrm{s}$. The results of computation are presented in the form of a pressure field and a velocity vector field on the shaft (Fig. 6 , 7). The lifting capacity of a pad with simple parallel holes with angled clearances located on the influence lines made up $3092 \mathrm{H}$, a pad with the Rayleigh angular clearances made up $3108 \mathrm{H}$.

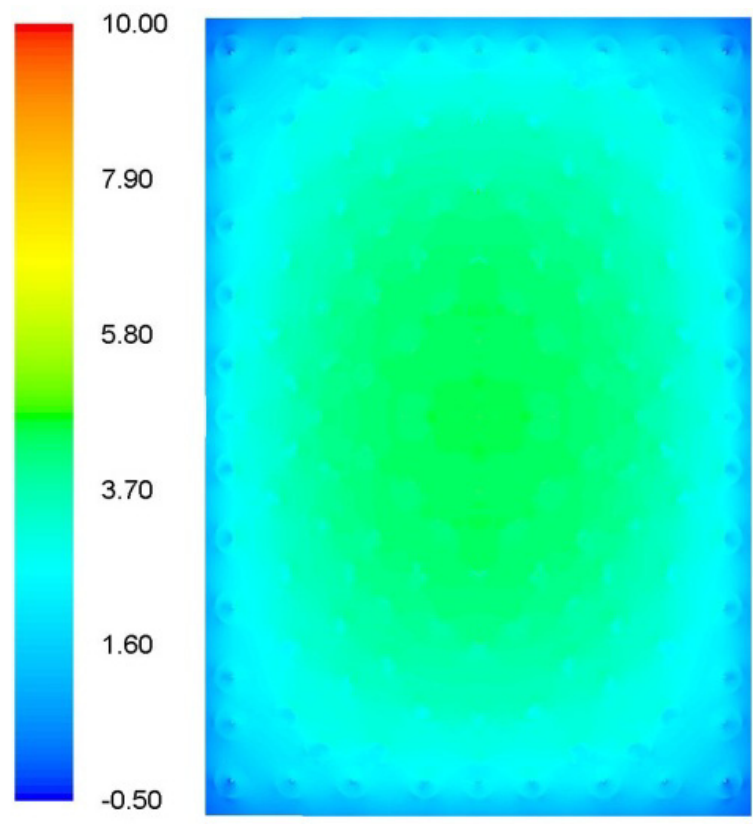

a)

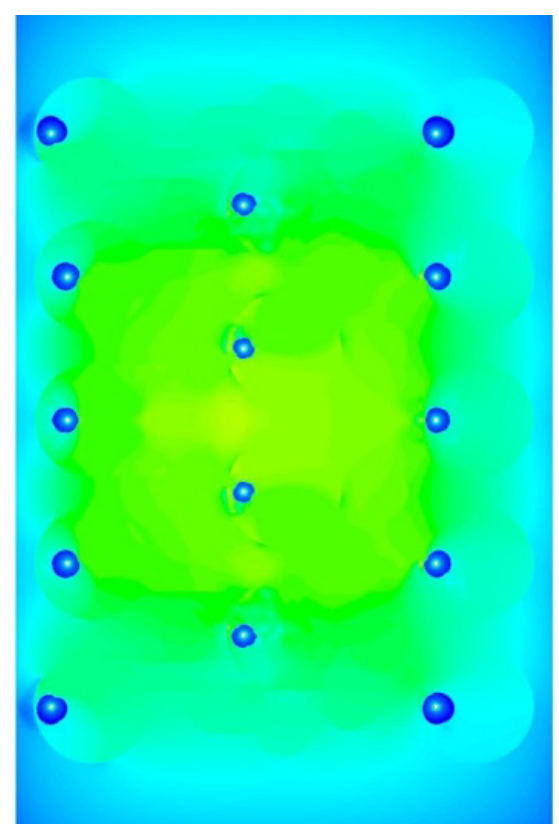

b)

Figure 6. Shaft pressure distribution : a) pad 1: $\mathrm{Q}=12.5 \mathrm{~g} / \mathrm{s}, \mathrm{F}=3092 \mathrm{H}$. b) pad 2: $\mathrm{Q}=12.5 \mathrm{~g} / \mathrm{s}, \mathrm{F}=3108 \mathrm{H}$. 


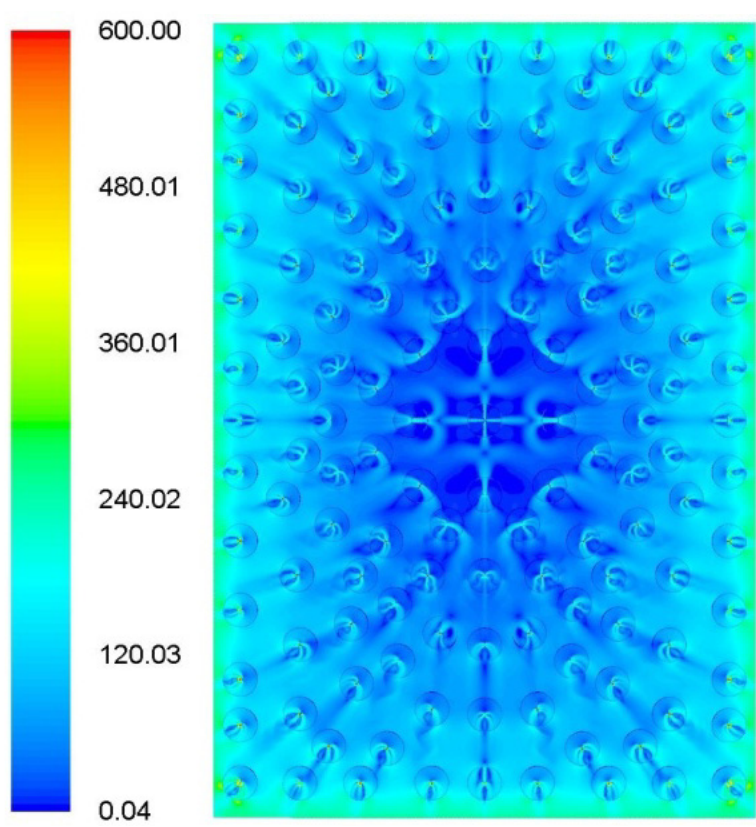

a)

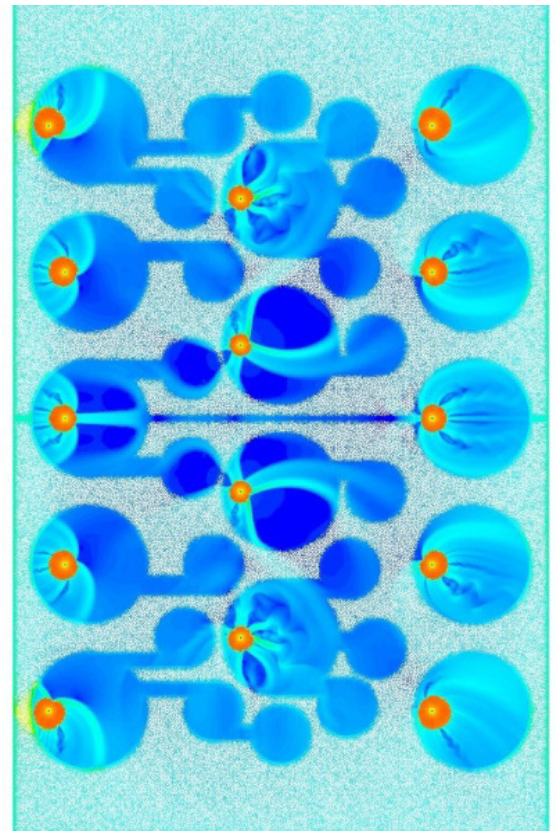

b)

Figure 7. Shaft velocity distribution: a) pad 1: $\mathrm{Q}=12.5 \mathrm{~g} / \mathrm{s}, \mathrm{F}=3092 \mathrm{H}$. b) pad 2: $\mathrm{Q}=12.5 \mathrm{~g} / \mathrm{s}, \mathrm{F}=3108 \mathrm{H}$

The developed pad with the Rayleigh angular clearances provides the lifting capacity a bit higher than for a pad with simple parallel holes with angular clearances located along the influence lines, which is the required result, as the hole number gets 9.5 times as little.

The pad 2 in Fig. 9 shows presence of overcritical efflux modes. This suggests nonoptimal relation of the rate and feed pressure of the working substance and the feeding nozzles area. It is necessary to continue perfecting the bearing surface geometry.

\section{Discussion}

The analysis of the gas bearing computation results demonstrates that, despite of considerable success in this field, the issues related to their central value remain burning.

The article covers the study of influence of such geometrical factors of a lubricating film as a diameter of distributing openings, a lubricating film thickness, a width of a target opening upon the lifting capacity. Computation of pads with a shaped lubricating film is given.

The study was carried out by means of solution of direct problems, i.e. proceeding from the given geometry and boundary conditions, as consequence the resultant force of the shaft pressure was defined. As against the majority of computations based on the Reynolds equation solution, for a solution of the delivered problems there was used the ANSYS Fluent (http://www.ansys.com) package, which solver uses the full Navier-Stokes equations.

The results of pad computation with a shaped lubricating film are presented. As the computation shows, a pad with smaller amount of openings and larger diameter, under its characteristics, can exceed a pad based on the principle of porous pads.

The pad changing load insensibility area is revealed, in which there can be dangerous vibration.

\section{Acknowledgments}

This article was prepared as part of the "1000 laboratories" program with the support of Saint-Petersburg National Research University of Information Technologies, Mechanics and Optics (University ITMO).

\section{References}

Beschastnykh, V. N. (2011). Development of a computation technique and experimental determination of characteristics of radial segment gas bearings for heavy rotors of GTU: theses of the dissertation of the 
PhD in technical sciences: 05.07.05, Moscow Aeronautical Institute, Moscow.

Bulat, M. P., \& Bulat, P. V. (2013). Basic classification of the gas-lubricated bearings. World Applied Sciences Journal, 28(10), 1444-1448. http://dx.doi.org/10.5829/idosi.wasj.2013.28.10.13924

Bulat, M. P., \& Bulat, P. V. (2013). The history of the gas bearings theory development. World Applied Sciences Journal, 27(7), 893-897. http://dx.doi.org/10.5829/idosi.wasj.2013.27.07.13718

Constantinescu, V. N. (1963). Lubrificatia cu gaze (633 p.). Bucuresti. Retrieved from http://www.worldcat.org/title/lubrificatia-cu-gaze/oclc/699569232

Gas lubricated sliding bearings [in Russian]. Under the edit. of S.A. Scheinberg, 2nd publ., revised and enlarged (1979). Moscow: Mashinostroenie, 336 p.

Gas-lubricated bearings [in Russian]. Under the edit. of N.S.Gressem, J.W.Powell (1966). Moscow: Mir, 415 p.

Ginzburg, I. P., Sokolov, E. I., \& Uskov, V. N. (1976). Types of wave patterns at interaction of an underexpanded jet with a plane endless barrier [in Russian]. Journal of Applied Mechanics and Technical Physics, 1, 45-50. http://www.sibran.ru/upload/iblock/4a1/4a17e37aeee4657c4dbf51db1487720e.pdf

Hydrostatic bearing designing [in Russian]. Under the edit. of Harry Rippel. Transl. from English by G.A.Andreeva (1967). Moscow: Mashinostroenie, 135 p.

Kosmynin, A. V. et al. (2011). Partially porous gas-static bearings of spindle assemblies. Theory and experiment [in Russian]. Moscow: Publishing House 'Academy of Natural Science', 126 p. ISBN: 978-5-7765-0774-8

Kotlyar, Ya. M. (1967). Asymptotic solutions of the Reynolds equation. Mechanics of fluids, 1, 161-165.

Loitsansky, L. G., \& Stepanyants, L. G. (1958). Hydrodynamical theory of spherical suspension [in Russian]. Works of Leningrad Polytechnic Institute, 198, 89-98.

Lord Rayleigh (1918). Notes on the theory of lubrication. Philosophical Magazine, 35(1), 1-12. http://dx.doi.org/10.1080/14786440108635730

Modern tribology. Results and prospects [in Russian]. Exec. Editor K.V. Frolov (2008), Moscow: Publishing House ZhI, 480 p. ISBN: 978-5-382-00518-8

O.Reynolds. (1886). On the theory of lubrication and its application to Mr. Beauchamp Tower's experiments, including an experimental determination of the viscosity of olive oil. Philosophical Transactions of the Royal Society of London, 177, 157-234. Retrieved from http:/www.jstor.org/discover/10.2307/109480?uid=3738936\&uid=2134\&uid=2\&uid=70\&uid=4\&sid=2110 4379474051

Robinson, C. H., \& Sterry, F. (1958). The static strength of pressure fed gas journal bearings: Jet bearings, AERE ED/R. - 1672, Harwell, Berkshire, England, 68. Retrieved from http:/www.worldcat.org/title/static-strength-of-pressure-fed-gas-journal-bearings-jet-bearings/oclc/8398302 3

Sipenkov, I. E. (1968). Construction of singular solutions for gas suspension with the help of a continuous boost scheme. Part of Bearing gas lubrication coll., Moscow: IMASh USSR Academy of Science, 41-48.

Stepanyants, L. G. (1967). Some methods of the gas-dynamic theory of lubrication [in Russian]. Works of Leningrad Polytechnic Institute, 280, 27-43.

Zablotsky, N. D. (1961). Linearization of boundary conditions in the theory of air suspension [in Russian]. Works of Leningrad Polytechnic Institute, 217, 127-132.

Zablotsky, N. D., Karyakin, V. E., \& Spienkov, I. E. (1970). Spherical gas bearing with forced boost [in Russian]. Mechanics of fluids, 3, 147-154.

\section{Copyrights}

Copyright for this article is retained by the author(s), with first publication rights granted to the journal.

This is an open-access article distributed under the terms and conditions of the Creative Commons Attribution license (http://creativecommons.org/licenses/by/3.0/). 